\title{
KP and Toda tau functions in Bethe ansatz
}

\author{
Kanehisa Takasaki \\ Graduate School of Human and Environmental Studies \\ Kyoto University \\ Yoshida, Sakyo, Kyoto, 606-8501, Japan \\ takasaki@math.h.kyoto-u.ac.jp
}

\begin{abstract}
Recent work of Foda and his group on a connection between classical integrable hierarchies (the KP and 2D Toda hierarchies) and some quantum integrable systems (the 6-vertex model with DWBC, the finite XXZ chain of spin 1/2, the phase model on a finite chain, etc.) is reviewed. Some additional information on this issue is also presented.

Keywords: six-vertex model; XXZ model; domain wall boundary condition; Bethe ansatz; KP hierarchy; Toda hierarchy; tau function
\end{abstract}

\section{Introduction}

Searching for a connection between classical and quantum integrable systems is an old and new subject, occasionally leading to a breakthrough towards a new area of research. One of the landmarks in this sense is the quantum inverse scattering method, also known as the algebraic Bethe ansatz. Stemming from the classical inverse scattering method, the algebraic Bethe ansatz covers a wide class of integrable systems including solvable models of statistical mechanics on the basis of the Yang-Baxter equations. ${ }^{1}$ Moreover, remarkably, it was recognized later that a kind of classical integrable systems (discrete Hirota equations) show up in the so called nested Bethe ansatz. $^{2}$

Recently a new connection was found by Foda and his group. ${ }^{3-7}$ They observed that special solutions of the classical integrable hierarchies (the $\mathrm{KP}$ and 2D Toda hierarchies) are hidden in quantum (or statistical) integrable systems such as the 6 -vertex model under the domain wall boundary condition (DWBC), ${ }^{3}$ the finite XXZ chain of spin $1 / 2,{ }^{4,5}$ and some other quantum integrable systems. ${ }^{6,7}$ Their results are based on a determinant formula of physical quantities, namely, the Izergin-Korepin formula for the 
partition function of the 6-vertex model ${ }^{8-10}$ and the Slavnov formula for the scalar product of Bethe states in the XXZ spin chain. ${ }^{11,12}$ Those formulae contain a set of free variables, and the determinant in the formula is divided by the Vandermonde determinant of these variables. Foda et al. interpreted the quotient of the determinant by the Vandermonde determinants as a tau function of the KP (or 2D Toda) hierarchy expressed in the so called "Miwa variables".

In this paper, we review these results along with some additional information on this issue. We are particularly interested in the relevance of the 2-component KP (2-KP) and 2D Toda hierarchies. Unfortunately, this research is still in an early stage, and we cannot definitely say which direction this research leads us to. A modest goal will be to understand the algebraic Bethe ansatz better in the perspective of classical integrable hierarchies.

This paper is organized as follows. In Section 2, we start with a brief account of the notion of Schur functions that play a fundamental role in the theory of integrable hierarchies, and introduce the tau function of the $\mathrm{KP}, 2-\mathrm{KP}$ and 2D Toda hierarchies as a function of both the usual time variables and the Miwa variables. Section 3 deals with the partition function of the 6 -vertex model with DWBC. Following the procedure of Foda et al., we rewrite the Izergin-Korepin formula into an almost rational form and show that a main part of the partition function can be interpreted as a KP tau function. Actually, the partition function allows two different interpretations that correspond to two choices of the Miwa variables. We examine a unified interpretation of the partition function as a tau function of the 2-KP (or 2D Toda) hierarchy. In Section 4, we turn to the finite XXZ chain of spin $1 / 2$, and present a similar interpretation to the scalar product of Bethe states (one of which depends on free variables) on the basis of the Slavnov formula. Section 5 is devoted to some other models including the phase model, ${ }^{13}$ which is also studied by the group of Foda. ${ }^{6}$ For those models, a determinant formula is known to hold for the scalar product of Bethe states both of which depend on free parameters. ${ }^{14}$ We consider a special case related to enumeration of boxed plane partitions.

\section{Tau functions}

\subsection{Schur functions}

Let us review the notion of Schur functions. We mostly follow the notations of Macdonald's book. ${ }^{15}$

For $N$ variables $\boldsymbol{x}=\left(x_{1}, \ldots, x_{N}\right)$ and a partition $\lambda=\left(\lambda_{1}, \lambda_{2}, \ldots, \lambda_{N}\right)$ 
$\left(\lambda_{1} \geq \lambda_{2} \geq \cdots \geq \lambda_{N} \geq 0\right)$ of length $(\lambda) \leq N$, the Schur function $s_{\lambda}(\boldsymbol{x})$ can be defined by Weyl's character formula

$$
s_{\lambda}(\boldsymbol{x})=\frac{\operatorname{det}\left(x_{j}^{\lambda_{i}-i+N}\right)_{i, j=1}^{N}}{\Delta(\boldsymbol{x})},
$$

where $\Delta(\boldsymbol{x})$ is the Vandermonde determinant

$$
\Delta(\boldsymbol{x})=\operatorname{det}\left(x_{j}^{-i+N}\right)_{i, j=1}^{N}=\prod_{1 \leq i<j \leq N}\left(x_{i}-x_{j}\right) .
$$

By one of the Jacobi-Trudi formulae, $s_{\lambda}(\boldsymbol{x})$ can be expressed as a determinant of the form

$$
s_{\lambda}(\boldsymbol{x})=\operatorname{det}\left(h_{\lambda_{i}-i+j}(\boldsymbol{x})\right)_{i, j=1}^{N},
$$

where $h_{n}(\boldsymbol{x}), n=0,1,2, \ldots$, are the completely symmetric functions

$$
h_{n}(\boldsymbol{x})=\sum_{1 \leq k_{1} \leq k_{2} \leq \cdots \leq k_{n} \leq N} x_{k_{1}} x_{k_{2}} \cdots x_{k_{n}} \quad \text { for } \quad n \geq 1, \quad h_{0}(\boldsymbol{x})=1 .
$$

The complete symmetric functions $h_{n}(\boldsymbol{x})$ themselves can be identified with the Schur functions for partitions with a single part:

$$
h_{n}(\boldsymbol{x})=s_{(n)}(\boldsymbol{x}), \quad(n):=(n, 0, \ldots, 0) .
$$

Another form of the Jacobi-Trudi formulae uses on the elementary symmetric functions $e_{n}(\boldsymbol{x})$. Since we shall not use it in the following, its details are omitted here.

The complete symmetric functions have the generating function

$$
\sum_{n=0}^{\infty} h_{n}(\boldsymbol{x}) z^{n}=\prod_{k=1}^{N}\left(1-x_{k} z\right)^{-1}=\exp \left(-\sum_{k=1}^{N} \log \left(1-x_{k} z\right)\right) .
$$

Since $\log \left(1-x_{k} z\right)$ has a Taylor expansion of the form

$$
\log \left(1-x_{k} z\right)=-\sum_{n=1}^{\infty} \frac{x_{k}^{n}}{n} z^{n}
$$

this generating function can be rewritten as

$$
\sum_{n=0}^{\infty} h_{n}(\boldsymbol{x}) z^{n}=\exp \left(\sum_{n=1}^{\infty} t_{n} z\right),
$$

where $t_{n}$ 's are defined as

$$
t_{n}=\frac{1}{n} \sum_{k=1}^{N} x_{k}^{n}
$$


In view of (2) and (3), one can redefine the complete symmetric functions and the Schur functions as functions $h_{n}[\boldsymbol{t}]$ and $s_{\lambda}[\boldsymbol{t}]{ }^{\mathrm{a}}$ of $\boldsymbol{t}=\left(t_{1}, t_{2}, \ldots\right)$, namely,

$$
\sum_{n=0}^{\infty} h_{n}[\boldsymbol{t}] z^{n}=\exp \left(\sum_{n=1}^{\infty} t_{n} z^{n}\right)
$$

and

$$
s_{\lambda}[\boldsymbol{t}]=\operatorname{det}\left(h_{\lambda_{i}-i+j}[\boldsymbol{t}]\right)_{i, j=1}^{N},
$$

where $\lambda$ is understood to be an infinite decreasing sequence $\lambda=\left(\lambda_{1}, \lambda_{2}, \ldots\right)$ with $\lambda_{i}=0$ for all but a finite number of $i$ 's, and $N$ is arbitrary integer greater than or equal to $l(\lambda)=\max \left\{i \mid \lambda_{i} \neq 0\right\}$. The right hand side of (6) is independent of $N$, because the lower left block of the matrix therein for $i>l(\lambda)$ and $j \leq l(\lambda)$ is zero and the lower right block for $i, j>l(\lambda)$ is an upper triangular matrix with 1 on the diagonal line.

This is a place where a connection with the KP hierarchy ${ }^{17}$ shows up. Namely, the variables $\boldsymbol{t}=\left(t_{1}, t_{2}, \ldots\right)$ are nothing but the "time variables" of the KP hierarchy, and the Schur functions $s_{\lambda}[\boldsymbol{t}]$ are special tau functions. As first pointed out by Miwa, ${ }^{18}$ viewing the tau function as a function of the $\boldsymbol{x}$ variables leads to a discrete (or difference) analogue of the KP hierarchy. For this reason, the $\boldsymbol{x}$ variables are sometimes referred to as "Miwa variables" in the literature of integrable systems.

\subsection{Tau functions of KP hierarchy}

Let us use the notation $\tau[\boldsymbol{t}]$ for the tau function in the usual sense (namely, a function of $\boldsymbol{t})$, and let $\tau(\boldsymbol{x})$ denote the function obtained from $\tau[\boldsymbol{t}]$ by the change of variables (4). It is the latter that plays a central role in this paper.

A general tau function of the KP hierarchy is a linear combination of the Schur functions

$$
\tau[\boldsymbol{t}]=\sum_{\lambda} c_{\lambda} s_{\lambda}[\boldsymbol{t}]
$$

where the coefficients $c_{\lambda}$ are Plücker coordinates of a point of an infinite dimensional Grassmann manifold (Sato Grassmannian). ${ }^{19}$ Roughly speaking, the Sato Grassmannian consists of linear subspaces $W \simeq \mathbf{C}^{\mathrm{N}}$ of a fixed

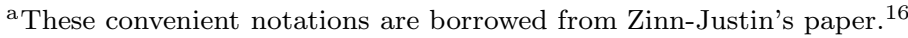


linear space $V \simeq \mathbf{C}^{\mathbf{Z}}$. We shall not pursue those fully general tau functions in the following.

We are interested in a smaller (but yet infinite dimensional) class of tau functions such that $c_{\lambda}=0$ for all partitions with $l(\lambda)>N$. This corresponds to a submanifold $\operatorname{Gr}(N, \infty)$ of the full Sato Grassmannian. The Plücker coordinates $c_{\lambda}$ are labelled by partitions of the form $\lambda=\left(\lambda_{1}, \ldots, \lambda_{N}\right)$, and given by finite determinants as

$$
c_{\lambda}=\operatorname{det}\left(f_{i, l_{j}}\right)_{i, j=1}^{N}, \quad l_{i}:=\lambda_{i}-i+N .
$$

Note that the sequences $\lambda_{i}$ 's and $l_{i}$ 's of non-negative integers are in one-toone correspondence:

$$
\infty>\lambda_{1} \geq \cdots \lambda_{N} \geq 0 \quad \longleftrightarrow \quad \infty>l_{1}>\cdots>l_{N} \geq 0 .
$$

The $N \times \infty$ matrix

$$
F=\left(f_{i j}\right)_{i=1, \ldots, N, j=0,1, \ldots}
$$

of parameters represent a point of the Grassmann manifold $\operatorname{Gr}(N, \infty)$.

By the Cauchy-Binet formula, the tau function $\tau(\boldsymbol{x})$ in the $\boldsymbol{x}$-picture can be expressed as

$$
\tau(\boldsymbol{x})=\sum_{\infty>l_{1}>\cdots>l_{N} \geq 0} \frac{\operatorname{det}\left(f_{i, l_{j}}\right)_{i, j=1}^{N} \operatorname{det}\left(x_{i}^{l_{j}}\right)_{i, j=1}^{N}}{\Delta(\boldsymbol{x})}=\frac{\operatorname{det}\left(f_{i}\left(x_{j}\right)\right)_{i, j=1}^{N}}{\Delta(\boldsymbol{x})},(9)
$$

where $f_{i}(x)$ 's are the power series of the form

$$
f_{i}(x)=\sum_{l=0}^{\infty} f_{i l} x^{l} .
$$

In particular, if there is a positive integer $M$ such that

$$
f_{i j}=0 \quad \text { for } \quad i \geq M+N
$$

(in other words, $f_{i}(x)$ 's are polynomials of degree less than $M+N$ ), the Plücker coordinate $c_{\lambda}$ vanishes for all Young diagrams not contained in the $N \times M$ rectangular Young diagram, namely,

$$
c_{\lambda}=0 \quad \text { for } \quad \lambda \nsubseteq\left(M^{N}\right):=(\underbrace{M, \ldots, M}_{N})
$$

The tau function $\tau[\boldsymbol{t}]$ thereby becomes a linear combination of a finite number of Schur function, hence a polynomial in $\boldsymbol{t}$. Geometrically, these solutions of the KP hierarchy sit on the finite dimensional Grassmann manifold $\operatorname{Gr}(N, N+M)$ of the Sato Grassmannian. 


\subsection{Tau functions of 2-KP hierarchy}

The tau function $\tau[\boldsymbol{t}, \overline{\boldsymbol{t}}]$ of the 2-component KP (2-KP) hierarchy is a function of two sequences $\boldsymbol{t}=\left(t_{1}, t_{2}, \ldots\right)$ and $\overline{\boldsymbol{t}}=\left(\bar{t}_{1}, \bar{t}_{2}, \ldots\right)$ of time variables, and can be expressed as

$$
\tau[\boldsymbol{t}, \overline{\boldsymbol{t}}]=\sum_{\lambda, \mu} c_{\lambda \mu} s_{\lambda}[\boldsymbol{t}] s_{\mu}[\overline{\boldsymbol{t}}],
$$

where $c_{\lambda \mu}$ 's are Plücker coordinates of a point of a 2-component analogue of the Sato Grassmannnian (which is, actually, isomorphic to the onecomponent version). ${ }^{19}$

The aforementioned class of tau functions of the KP hierarchy can be generalized to the 2-component case. Such tau functions correspond to points of the submanifold $\operatorname{Gr}(M+N, 2 \infty)$ of the 2-component Sato Grassmannian. For those tau functions, the Plücker coordinates $c_{\lambda \mu}$ vanish if $l(\lambda)>M$ or $l(\mu)>N$; the remaining Plücker coordinates are given by finite determinants of a matrix with two rectangular blocks of size $(M+N) \times M$ and $(M+N) \times N$ as

$$
c_{\lambda \mu}=\operatorname{det}\left(f_{i, l_{j}} \mid g_{i, m_{k}}\right) \text {, }
$$

where $i$ is the row index ranging over $i=1, \ldots, M+N$ and $j, k$ are column indices in the two blocks ranging over $j=1, \ldots, M$ and $k=1, \ldots, N$, respectively. $l_{j}$ 's and $m_{k}$ 's are related to the parts of $\lambda=\left(\lambda_{j}\right)_{j=1}^{M}$ and $\mu=\left(\mu_{i}\right)_{j=1}^{N}$ as

$$
l_{j}=\lambda_{j}-j+M, \quad m_{k}=\mu_{k}-k+N .
$$

By the change of variables from $\boldsymbol{x}$ and $\boldsymbol{y}$ to

$$
t_{n}=\frac{1}{n} \sum_{j=1}^{M} x_{j}^{n}, \quad \bar{t}_{n}=\frac{1}{n} \sum_{k=1}^{N} y_{k}^{n},
$$

the tau function $\tau[\boldsymbol{t}, \overline{\boldsymbol{t}}]$ is converted to the $(\boldsymbol{x}, \boldsymbol{y})$-picture $\tau(\boldsymbol{x}, \boldsymbol{y})$. Again by the Cauchy-Binet formula, $\tau(\boldsymbol{x}, \boldsymbol{y})$ turns out to be a quotient of two determinants as

$$
\tau(\boldsymbol{x}, \boldsymbol{y})=\frac{\operatorname{det}\left(f_{i}\left(x_{j}\right) \mid g_{i}\left(y_{k}\right)\right)}{\Delta(\boldsymbol{x}) \Delta(\boldsymbol{y})},
$$

where the denominator is the determinant with the same block structure as $(11)$, and $f_{i}(x)$ and $g_{j}(y)$ are power series of the form

$$
f_{i}(x)=\sum_{l=0}^{\infty} f_{i l} x^{l}, \quad g_{i}(y)=\sum_{l=0}^{\infty} g_{i l} y^{l} .
$$




\subsection{Tau function of $2 D$ Toda hierarchy}

The 2-KP hierarchy is closely related to the $2 \mathrm{D}$ Toda hierarchy. ${ }^{20}$ The tau function $\tau_{s}[\boldsymbol{t}, \overline{\boldsymbol{t}}]$ of the 2D Toda hierarchy depends on a discrete variable (lattice coordinate) $s$ alongside the two series of time variables $\boldsymbol{t}$ and $\overline{\boldsymbol{t}}$. For each value of $s, \tau_{s}[\boldsymbol{t}, \overline{\boldsymbol{t}}]$ is a tau function of the 2-KP hierarchy, and these 2-KP tau functions are mutually connected by a kind of Bäcklund transformations. Consequently, $\tau_{s}[\boldsymbol{t}, \overline{\boldsymbol{t}}]$ can be expressed as shown in (10) with the coefficients $c_{s \lambda \mu}$ depending on $s$.

Actually, it is more natural to use $s_{\lambda}[\boldsymbol{t}] s_{\mu}[-\overline{\boldsymbol{t}}]$ rather than $s_{\lambda}[\boldsymbol{t}] s_{\mu}[\overline{\boldsymbol{t}}]$ for the Schur function expansion of the Toda tau function. ${ }^{21}$ (Note that $s_{\mu}[-\bar{t}]$ can be rewritten as

$$
s_{\mu}[-\overline{\boldsymbol{t}}]=(-1)^{|\mu|} s_{{ }_{\mu}}[\boldsymbol{t}],
$$

where ${ }^{\mathrm{t}} \mu$ denotes the transpose of $\mu$.) Expanded in these product of tau functions as

$$
\tau_{s}[\boldsymbol{t}, \overline{\boldsymbol{t}}]=\sum_{\lambda, \mu} c_{s \lambda \mu} s_{\lambda}[\boldsymbol{t}] s_{\mu}[-\overline{\boldsymbol{t}}]
$$

the coefficients $c_{s \lambda \mu}$ become Plücker coordinates of an infinite dimensional flag manifold. Intuitively, they are minor determinants

$$
c_{s \lambda \mu}=\operatorname{det}\left(g_{\lambda_{i}-i+s, \mu_{j}-j+s}\right)_{i, j=1}^{\infty}
$$

of an infinite matrix $g=\left(g_{i j}\right)_{i, j \in \mathbf{Z}}$, though this definition requires justification. ${ }^{21}$ In particular, if $g$ is a diagonal matrix, the coefficients $c_{s \lambda \mu}$ are also diagonal (namely, $c_{s \lambda \mu} \propto \delta_{\lambda \mu}$ ) and the Schur function expansion (14) simplifies to the "diagonal" form

$$
\tau_{s}[\boldsymbol{t}, \overline{\boldsymbol{t}}]=\sum_{\lambda} c_{s \lambda} s_{\lambda}[\boldsymbol{t}] s_{\lambda}[-\overline{\boldsymbol{t}}], \quad c_{s \lambda}=\prod_{i=1}^{\infty} g_{\lambda_{i}-i+s} .
$$

If we reformulate the $2 \mathrm{D}$ Toda hierarchy on the semi-infinite lattice $s \geq 0$, the infinite determinants defining $c_{\lambda}$ 's are replaced by finite determinants, and $\tau_{s}[\boldsymbol{t}, \overline{\boldsymbol{t}}]$ itself becomes a finite determinant. We shall encounter an example of such tau functions in the next section.

\section{6-vertex model with DWBC}

\subsection{Setup of model}

We consider the 6-vertex model on an $N \times N$ square lattice with inhomogeneity parameters $\boldsymbol{u}=\left(u_{1}, \ldots, u_{N}\right)$ and $\boldsymbol{v}=\left(v_{1}, \ldots, v_{N}\right)$ assigned to 


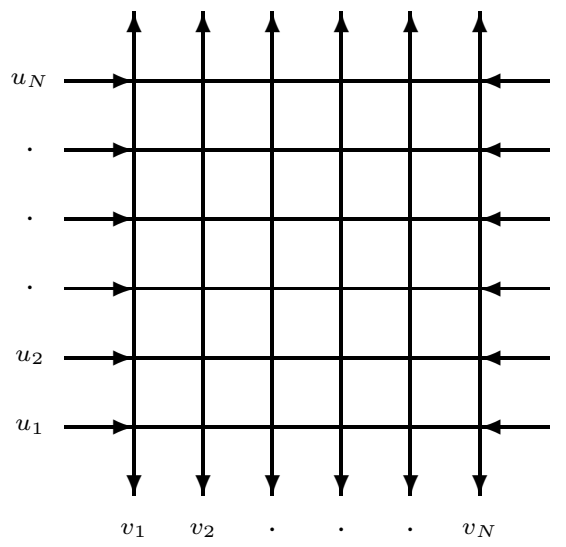

Fig. 1. Square lattice with DWBC

the rows and columns. The boundary of the lattice is supplemented with extra edges pointing outwards, and the domain-wall boundary condition (DWBC) is imposed on these extra edges. Namely, the arrows on the extra edges on top and bottom of the boundary are pointing outwards, and those on the other extra edges are pointing inwards (see figure 1).

The vertex at the intersection of the $i$-th row and the $j$-th column is given the following weight $w_{i j}$ determined by the configuration of arrows on the adjacent edges:

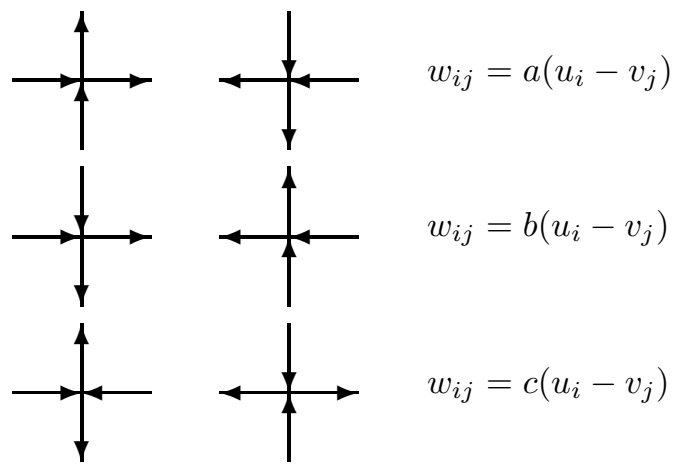

The weight functions $a(u), b(u), c(u)$ are defined as

$$
a(u)=\sinh (u+\gamma), \quad b(u)=\sinh u, \quad c(u)=\sinh \gamma,
$$

where $\gamma$ is a parameter. Thus the partition function of this model is defined 
as a function of the inhomogeneity parameters $\boldsymbol{u}$ and $\boldsymbol{v}$ :

$$
Z_{N}=Z_{N}(\boldsymbol{u}, \boldsymbol{v})=\sum_{\text {configuration }} \prod_{i, j=1}^{N} w_{i j} .
$$

\subsection{Izergin-Korepin formula for $Z_{N}$}

According to the result of Korepin ${ }^{8}$ and Izergin, ${ }^{9}$ the partition function $Z_{N}$ has the determinant formula

$$
\begin{gathered}
Z_{N}=\frac{\prod_{i, j=1}^{N} \sinh \left(u_{i}-v_{j}+\gamma\right) \sinh \left(u_{i}-v_{j}\right)}{\prod_{1 \leq i<j \leq N} \sinh \left(u_{i}-u_{j}\right) \sinh \left(v_{j}-v_{i}\right)} \\
\quad \times \operatorname{det}\left(\frac{\sinh \gamma}{\sinh \left(u_{i}-v_{j}+\gamma\right) \sinh \left(u_{i}-v_{j}\right)}\right)_{i, j=1}^{N},
\end{gathered}
$$

which one can rewrite as

$$
\begin{aligned}
Z_{N}=\frac{\sinh ^{N} \eta}{\prod_{1 \leq i<j \leq N} \sinh \left(u_{i}-u_{j}\right) \sinh \left(v_{j}-v_{i}\right)} \\
\quad \times \operatorname{det}\left(\frac{\prod_{k=1}^{N} \sinh \left(u_{i}-v_{k}+\gamma\right) \sinh \left(u_{i}-v_{k}\right)}{\sinh \left(u_{i}-v_{j}+\gamma\right) \sinh \left(u_{i}-v_{j}\right)}\right)_{i, j=1}^{N}
\end{aligned}
$$

and

$$
\begin{aligned}
Z_{N}=\frac{\sinh ^{N} \gamma}{\prod_{1 \leq i<j \leq N} \sinh \left(u_{i}-u_{j}\right) \sinh \left(v_{j}-v_{i}\right)} \\
\quad \times \operatorname{det}\left(\frac{\prod_{k=1}^{N} \sinh \left(u_{k}-v_{j}+\gamma\right) \sinh \left(u_{k}-v_{j}\right)}{\sinh \left(u_{i}-v_{j}+\gamma\right) \sinh \left(u_{i}-v_{j}\right)}\right)_{i, j=1}^{N} .
\end{aligned}
$$

If we introduce the new variables and parameters ${ }^{3}$

$$
x_{i}:=e^{2 u_{i}}, \quad y_{i}:=e^{2 v_{i}}, \quad q:=e^{-\gamma},
$$

we can rewrite these formulae as

$$
\begin{aligned}
Z_{N}=C_{N} \prod_{i, j=1}^{N}( & \left.x_{i} q^{-1}-y_{j} q\right)\left(x_{i}-y_{j}\right) \\
& \quad \times \frac{1}{\Delta(\boldsymbol{x}) \Delta(\boldsymbol{y})} \operatorname{det}\left(\frac{q^{-1}-q}{\left(x_{i} q^{-1}-y_{j} q\right)\left(x_{i}-y_{j}\right)}\right)_{i, j=1}^{N},
\end{aligned}
$$




$$
Z_{N}=\frac{C_{N}\left(q^{-1}-q\right)^{N}}{\Delta(\boldsymbol{x}) \Delta(\boldsymbol{y})} \operatorname{det}\left(\frac{\prod_{k=1}^{N}\left(x_{i} q^{-1}-y_{k} q\right)\left(x_{i}-y_{k}\right)}{\left(x_{i} q^{-1}-y_{j} q\right)\left(x_{i}-y_{j}\right)}\right)_{i, j=1}^{N}
$$

and

$$
Z_{N}=\frac{C_{N}\left(q^{-1}-q\right)^{N}}{\Delta(\boldsymbol{x}) \Delta(\boldsymbol{y})} \operatorname{det}\left(\frac{\prod_{k=1}^{N}\left(x_{k} q^{-1}-y_{j} q\right)\left(x_{k}-y_{j}\right)}{\left(x_{i} q^{-1}-y_{j} q\right)\left(x_{i}-y_{j}\right)}\right)_{i, j=1}^{N}
$$

where $C_{N}=C_{N}(\boldsymbol{u}, \boldsymbol{v})$ is an exponential function of a linear combination of $u_{i}$ 's and $v_{i}$ 's. Apart from this simple factor, $Z_{N}$ thus reduces to a rational function of $\boldsymbol{x}=\left(x_{1}, \ldots, x_{N}\right)$ and $\boldsymbol{y}=\left(y_{1}, \ldots, y_{N}\right)$.

\section{3. $K P$ and 2-KP tau functions hidden in $Z_{N}$}

As pointed out by Foda et al., ${ }^{3}$ two KP tau functions are hidden in these determinant formulae of $Z_{N}$. Firstly, if $y_{i}$ 's are considered to be constants, the $\boldsymbol{x}$-dependent part of (22) give the function

$$
\tau_{1}(\boldsymbol{x})=\frac{\operatorname{det}\left(f_{j}\left(x_{i}\right)\right)_{i, j=1}^{N}}{\Delta(\boldsymbol{x})}, \quad f_{j}(x):=\frac{\prod_{k=1}^{N}\left(x q^{-1}-y_{k} q\right)\left(x-y_{k}\right)}{\left(x q^{-1}-y_{j} q\right)\left(x-y_{j}\right)} .
$$

This is a tau function of the KP hierarchy with respect to $t_{n}=\frac{1}{n} \sum_{k=1}^{N} x_{k}^{n}$. Moreover, since $f_{j}(x)$ 's are polynomials in $x$, this tau function is as a polynomial in $\boldsymbol{t}$. In the same sense, if $x_{i}$ 's are considered to be constants, the $\boldsymbol{y}$-dependent part of (23) gives the function

$$
\tau_{2}(\boldsymbol{y})=\frac{\operatorname{det}\left(g_{i}\left(y_{j}\right)\right)_{i, j=1}^{N}}{\Delta(\boldsymbol{y})}, \quad g_{i}(y):=\frac{\prod_{k=1}^{N}\left(x_{i} q^{-1}-y q\right)\left(x_{i}-y\right)}{\left(x_{i} q^{-1}-y q\right)\left(x_{i}-y\right)},
$$

which is a polynomial tau function of the KP hierarchy with respect to $\bar{t}_{n}=\frac{1}{n} \sum_{k=1}^{N} y_{k}^{n}$.

Thus, apart from an irrelevant factor, $Z_{N}$ is a tau function of the KP hierarchy with respect to $\boldsymbol{x}$ and $\boldsymbol{y}$ separately. It will be natural to ask whether a tau function of the 2-KP hierarchy is hidden in $Z_{N}$.

A partial answer can be found in the work of Stroganov ${ }^{22}$ and Okada. ${ }^{23}$ According to their results, if $q=e^{\pi i / 3}$, the partition function coincides, up to a simple factor, with a single Schur function of $(\boldsymbol{x}, \boldsymbol{y})$ as

$$
Z_{N}=(\text { simple factor }) s_{\lambda}(\boldsymbol{x}, \boldsymbol{y}),
$$

where $\lambda$ is the double staircase partition

$$
\lambda=(N-1, N-1, N-2, N-2, \ldots, 1,1)
$$


of length $2 N$. By the way, for any partition $\lambda=\left(\lambda_{1}, \ldots, \lambda_{2 N}\right)$ of length $\leq 2 N$, the Weyl character formula for $s_{\lambda}(\boldsymbol{x}, \boldsymbol{y})$ reads

$$
s_{\lambda}(\boldsymbol{x}, \boldsymbol{y})=\frac{\operatorname{det}\left(x_{j}^{\lambda_{i}-i+2 N} \mid y_{k}^{\lambda_{i}-i+2 N}\right)}{\Delta(\boldsymbol{x}, \boldsymbol{y})},
$$

where the row index $i$ ranges over $i=1, \ldots, 2 N$ and the column indices $j, k$ in the two blocks over $j, k=1, \ldots, N$. Multiplying this function by $\Delta(\boldsymbol{x}, \boldsymbol{y}) / \Delta(\boldsymbol{x}) \Delta(\boldsymbol{y})$ gives

$$
\frac{\Delta(\boldsymbol{x}, \boldsymbol{y})}{\Delta(\boldsymbol{x}) \Delta(\boldsymbol{y})} s_{\lambda}(\boldsymbol{x}, \boldsymbol{y})=\frac{\operatorname{det}\left(x_{j}^{\lambda_{i}-i+2 N} \mid y_{k}^{\lambda_{i}-i+2 N}\right)}{\Delta(\boldsymbol{x}) \Delta(\boldsymbol{y})},
$$

which may be thought of as a 2-KP tau function of the form (13).

Another answer, which is valid for arbitrary values of $q$, was found by Zinn-Justin (private communication). Let us note that (21) can be rewritten as

$$
\begin{gathered}
Z_{N}=C_{N}\left(q^{-1}-q\right)^{N} \prod_{i, j=1}^{N}\left(1-q^{-2} x_{i} y_{j}^{-1}\right)\left(1-x_{i} y_{j}^{-1}\right) \prod_{1 \leq i<j \leq N}\left(-y_{i} y_{j}\right) \\
\times \frac{1}{\Delta(\boldsymbol{x}) \Delta\left(\boldsymbol{y}^{-1}\right)} \operatorname{det}\left(\frac{1}{\left(1-q^{-2} x_{i} y_{j}^{-1}\right)\left(1-x_{i} y_{j}^{-1}\right)}\right)_{i, j=1}^{N},
\end{gathered}
$$

where

$$
\boldsymbol{y}^{-1}=\left(y_{1}^{-1}, \ldots, y_{N}^{-1}\right) .
$$

The last part of thins expression, namely the quotient of the determinant by the Vandermonde determinants $\Delta(\boldsymbol{x}) \Delta\left(\boldsymbol{y}^{-1}\right)$, may be thought of as a 2 -KP tau function with respect to the time variables

$$
t_{n}=\frac{1}{n} \sum_{k=1}^{N} x_{k}^{n}, \quad \bar{t}_{n}=-\frac{1}{n} \sum_{k=1}^{N} y_{k}^{-n} .
$$

This is a special case of the tau functions

$$
\tau(\boldsymbol{x}, \boldsymbol{y})=\frac{\operatorname{det}\left(h\left(x_{i} y_{j}^{-1}\right)\right)_{i, j=1}^{N}}{\Delta(\boldsymbol{x}) \Delta\left(\boldsymbol{y}^{-1}\right)},
$$

considered by Orlov and Shiota, ${ }^{24}$ where $h(z)$ is an arbitrary power series of the form

$$
h(z)=\sum_{n=0}^{\infty} h_{n} z^{n}, \quad h_{n} \neq 0 \quad \text { for } \quad n \geq 0 .
$$


By the Cauchy-Binet formula, $\tau(\boldsymbol{x}, \boldsymbol{y})$ can be expanded as

$$
\tau(\boldsymbol{x}, \boldsymbol{y})=\sum_{\lambda=\left(\lambda_{1}, \ldots, \lambda_{N}\right)} c_{\lambda} s_{\lambda}(\boldsymbol{x}) s_{\lambda}\left(\boldsymbol{y}^{-1}\right), \quad c_{\lambda}:=\prod_{i=1}^{N} h_{\lambda_{i}-i+N} .
$$

This is an analogue (for a semi-infinite lattice) of the Toda tau functions of the diagonal form (16). Note that the role of the lattice coordinate $s$ is played by $N$. Since the number of the Miwa variables in (29) also depends on $N$, translation to the lanugage of the 2D Toda hierarchy is somewhat tricky, but this tricky situation is rather common in random matrix models. ${ }^{24}$ Thus, though not of the type shown in (13), the last part of (28) turns out to be a $2-\mathrm{KP}$ tau function.

Lastly, let us mention that Korepin and Zinn-Justin considered the partition function in the homogeneous limit as $u_{i}, v_{j} \rightarrow 0 .{ }^{25}$ In that limit, the partition function reduces, up to a simple factor, to a special tau function of the 1D Toda equation, and can be treated as an analogue of random matrix models.

\section{Scalar product of states in finite $\mathrm{XXZ}$ spin chain}

\subsection{L- and $T$-matrices for spin $1 / 2$ chain}

We consider a finite XXZ spin chain of spin $1 / 2$ and length $N$ with inhomongeneity parameters $\xi_{l}, l=1, \ldots, N$. To define local $L$-matrices, let us introduce the $2 \times 2$ matrix $L(u)=\left(L_{i j}(u)\right)_{i, j=1,2}$ of the $2 \times 2$ blocks

$$
\begin{aligned}
& L_{11}(u)=a(u) \frac{1+\sigma^{3}}{2}+b(u) \frac{1-\sigma^{3}}{2}, \quad L_{12}(u)=c(u) \sigma^{-}, \\
& L_{21}(u)=c(u) \sigma^{+}, \quad L_{22}(u)=b(u) \frac{1+\sigma^{3}}{2}+a(u) \frac{1-\sigma^{3}}{2},
\end{aligned}
$$

where $\sigma^{ \pm}$and $\sigma^{3}$ are the Pauli matrices

$$
\sigma^{+}=\left(\begin{array}{ll}
0 & 1 \\
0 & 0
\end{array}\right), \quad \sigma^{-}=\left(\begin{array}{ll}
0 & 0 \\
1 & 0
\end{array}\right), \quad \sigma^{3}=\left(\begin{array}{cc}
1 & 0 \\
0 & -1
\end{array}\right) .
$$

These $2 \times 2$ blocks are understood to act on the single spin space $\mathbf{C}^{2}$. The structure functions $a(u), b(u), c(u)$ are the same as the weight functions $(17)$ for the 6 -vertex model, and built into the $R$-matrix

$$
R(u-v)=\left(\begin{array}{cccc}
a(u-v) & 0 & 0 & 0 \\
0 & b(u-v) & c(u-v) & 0 \\
0 & c(u-v) & b(u-v) & 0 \\
0 & 0 & 0 & a(u-v)
\end{array}\right) .
$$


Let $L^{(l)}\left(u-\xi_{l}\right)=\left(L_{i j}^{(l)}(u-\xi)\right)_{i, j=1,2}$ be the local $L$-matrix at the $l$-th site,

$$
L_{i j}^{(l)}\left(u-\xi_{l}\right)=\cdots \otimes 1 \otimes L_{i j}\left(u-\xi_{l}\right) \otimes 1 \otimes \cdots,
$$

and define the $T$-matrix as

$$
T(u)=\left(\begin{array}{ll}
A(u) & B(u) \\
C(u) & D(u)
\end{array}\right)=L^{(1)}\left(u-\xi_{1}\right) \cdots L^{(N)}\left(u-\xi_{N}\right) .
$$

The matrix elements of these matrices, hence the trace of the $T$-matrix

$$
\mathcal{T}(u)=\operatorname{Tr} T(u)=A(u)+D(u)
$$

as well, are operators on the full spin space $V=\bigotimes_{l=1}^{N} \mathbf{C}^{2}$. The $L$-matrices satisfy the local intertwining relations

$$
\begin{aligned}
R(u-v)\left(L^{(l)}(u) \otimes I\right)\left(I \otimes L^{(m)}(v)\right) & \\
& =\left(I \otimes L^{(m)}(v)\right)\left(L^{(l)}(u) \otimes I\right) R(u-v),
\end{aligned}
$$

where $R(u-v), L^{(l)}(u) \otimes I$ and $I \otimes K^{(m)}(v)$ are understood to be $4 \times 4$ matrices (of scalars or of spin operators on $V$ ) acting on the tensor product $\mathbf{C}^{2} \otimes \mathbf{C}^{2}$ of two copies of the auxiliary space $\mathbf{C}^{2}$. These local intertwining relations lead to the global intertwining relation

$$
R(u-v)(T(u) \otimes I)(I \otimes T(v))=(I \otimes T(v))(T(u) \otimes I) R(u-v)
$$

for the $T$-matrix. This is a compact expression of many bilinear relations among the matrix elements of $T(u)$ and $T(v)$, such as

$$
\begin{aligned}
& A(u) B(v)=f(u-v) B(v) A(u)-g(u-v) B(u) A(v), \\
& D(u) B(v)=f(u-v) B(v) D(u)-g(u-v) B(u) D(v), \\
& C(u) B(v)=g(u-v)(A(u) D(v)-A(v) D(u))
\end{aligned}
$$

and

$$
\begin{aligned}
& {[A(u), A(v)]=0, \quad[B(u), B(v)]=0,} \\
& {[C(u), C(v)]=0, \quad[D(u), D(v)]=0,}
\end{aligned}
$$

where

$$
f(u)=\frac{a(u)}{b(u)}=\frac{\sinh (u+\gamma)}{\sinh u}, \quad g(u)=\frac{c(u)}{b(u)}=\frac{\sinh \gamma}{\sinh u} .
$$

A consequence of those relations is the fact that $\mathcal{T}(u)$ and $\mathcal{T}(v)$ commute for any values of $u, v$ :

$$
[\mathcal{T}(u), \mathcal{T}(v)]=0 .
$$

The algebraic Bethe ansatz is a method for constructing simultaneous eigenstates (called "Bethe states") of $\mathcal{T}(u)$ for all values of $u$. 


\subsection{Algebraic Bethe ansatz}

Let us introduce the pseudo-vacuum 0$\rangle$ and its dual $\langle 0|$ :

$$
\left\langle 0\left|=\bigotimes_{l=1}^{N}\left(\begin{array}{ll}
1 & 0
\end{array}\right) \in V^{*}, \quad\right| 0\right\rangle=\bigotimes_{l=1}^{N}\left(\begin{array}{l}
1 \\
0
\end{array}\right) \in V .
$$

They indeed satisfy the vacuum conditions

$$
\begin{array}{ll}
\langle 0| B(u)=0, & C(u)|0\rangle=0, \\
A(u)|0\rangle=\alpha(u)|0\rangle, & D(u)|0\rangle=\delta(u)|0\rangle, \\
\langle 0| A(u)=\alpha(u)\langle 0|, & \langle 0| D(u)=\delta(u)\langle 0|,
\end{array}
$$

where

$$
\alpha(u)=\prod_{l=1}^{N} \sinh \left(u-\xi_{l}+\gamma\right), \quad \delta(u)=\prod_{l=1}^{N} \sinh \left(u-\xi_{l}\right) .
$$

For notational convenience, we introduce the reflection coefficients

$$
r(u)=\frac{\alpha(u)}{\delta(u)} .
$$

Bethe states are generated from $|0\rangle$ by the action of $B\left(v_{j}\right)$ 's. Suppose that $v_{j}$ 's satisfy the Bethe equations

$$
r\left(v_{i}\right) \prod_{j \neq i} \frac{\sinh \left(v_{i}-v_{j}-\gamma\right)}{\sinh \left(v_{i}-v_{j}+\gamma\right)}=1, \quad i=1, \ldots, n .
$$

The state $\prod_{i=1}^{n} B\left(v_{i}\right)|0\rangle$ then becomes an eigenstate of $\mathcal{T}(u)$ :

$$
\begin{aligned}
\mathcal{T}(u) \prod_{i=1}^{n} B\left(v_{i}\right)|0\rangle & \\
& =\left(\alpha(u) \prod_{i=1}^{n} f\left(v_{i}-u\right)+\delta(u) \prod_{i=1}^{n} f\left(u-v_{i}\right)\right) \prod_{i=1}^{n} B\left(v_{i}\right)|0\rangle .
\end{aligned}
$$

Let us remark that the operators $A(u), B(u), C(u), D(u)$ are related to a row-to-row transfer matrix of the 6 -vertex model on the square lattice. One can thereby derive the identities ${ }^{10}$

$$
\begin{aligned}
& \langle 0| \prod_{i=1}^{N} C\left(u_{i}\right)=\langle\overline{0}| Z_{N}\left(u_{1}, \ldots, u_{N}, \xi_{1}, \ldots, \xi_{N}\right), \\
& \prod_{i=1}^{N} B\left(u_{i}\right)|0\rangle=Z_{N}\left(u_{1}, \ldots, u_{N}, \xi_{1}, \ldots, \xi_{N}\right)|\overline{0}\rangle,
\end{aligned}
$$


where $u_{i}$ 's are free (namely, not required to satisfy the Bethe equations) variables, and $|\overline{0}\rangle$ and $\langle\overline{0}|$ denote the "anti-pseudo-vacuum" and its dual:

$$
\left\langle\overline{0}\left|=\bigotimes_{l=1}^{N}\left(\begin{array}{ll}
0 & 1
\end{array}\right) \in V^{*}, \quad\right| \overline{0}\right\rangle=\bigotimes_{l=1}^{N}\left(\begin{array}{l}
0 \\
1
\end{array}\right) \in V .
$$

\subsection{Slavnov formula for scalar product}

Let $\boldsymbol{u}=\left(u_{1}, \ldots, u_{n}\right)$ be free variables and $\boldsymbol{v}=\left(v_{1}, \ldots, v_{n}\right)$ satisfy the Bethe equations (38). The Slavnov formula ${ }^{11,12}$ for the scalar product

$$
S_{n}(\boldsymbol{u}, \boldsymbol{v})=\left\langle 0\left|\prod_{i=1}^{n} C\left(u_{i}\right) \prod_{i=1}^{n} B\left(v_{i}\right)\right| 0\right\rangle
$$

reads

$$
S_{n}(\boldsymbol{u}, \boldsymbol{v})=\frac{\prod_{i=1}^{n} \delta\left(u_{i}\right) \delta\left(v_{i}\right) \prod_{i, j=1}^{n} \sinh \left(u_{i}-v_{j}+\gamma\right)}{\prod_{1 \leq i<j \leq n} \sinh \left(u_{i}-u_{j}\right) \sinh \left(v_{j}-v_{i}\right)} \operatorname{det}\left(H_{i j}\right)_{i, j=1}^{n},
$$

where

$$
H_{i j}=\frac{\sinh \gamma}{\sinh \left(u_{i}-v_{j}+\gamma\right) \sinh \left(u_{i}-v_{j}\right)}\left(1-r\left(u_{i}\right) \prod_{k \neq i} \frac{\sinh \left(u_{i}-v_{k}-\gamma\right)}{\sinh \left(u_{i}-v_{k}+\gamma\right)}\right) .
$$

One can rewrite this formula as

$$
S_{n}(\boldsymbol{u}, \boldsymbol{v})=\frac{\sinh ^{n} \gamma \prod_{i=1}^{n} \delta\left(v_{i}\right)}{\prod_{1 \leq i<j \leq n} \sinh \left(u_{i}-u_{j}\right) \sinh \left(v_{j}-v_{i}\right)} \operatorname{det}\left(K_{i j}\right)_{i, j=1}^{n},
$$

where

$$
K_{i j}=\frac{\delta\left(u_{i}\right) \prod_{k \neq j} \sinh \left(u_{i}-v_{k}+\gamma\right)-\alpha\left(u_{i}\right) \prod_{k \neq j} \sinh \left(u_{i}-v_{k}-\gamma\right)}{\sinh \left(u_{i}-v_{j}\right)} .
$$

\subsection{KP tau function hidden in $S_{n}(u, v)$}

If we introduce the new variables and parameters ${ }^{4}$

$$
x_{i}:=e^{2 u_{i}}, \quad y_{i}:=e^{2 v_{i}}, \quad z_{i}:=e^{2 \xi_{i}}, \quad q:=e^{-\gamma},
$$

the Slavnov formula can be converted to the almost rational form

$$
S_{n}(\boldsymbol{u}, \boldsymbol{v})=\frac{C_{n} \sinh ^{n} \gamma \prod_{i=1}^{n} \delta\left(v_{i}\right)}{\Delta(\boldsymbol{y})} \frac{\operatorname{det}\left(f_{j}\left(x_{i}\right)\right)_{i, j=1}^{n}}{\Delta(\boldsymbol{x})},
$$


where $C_{n}=C_{n}(\boldsymbol{u}, \boldsymbol{v})$ is an exponential function of a linear combination of $u_{i}$ 's and $v_{i}$ 's, and

$$
f_{j}(x)=\frac{\prod_{l=1}^{N}\left(x-z_{l}\right) \prod_{k \neq j}\left(q^{-1} x-q y_{k}\right)-\prod_{l=1}^{N}\left(q^{-1} x-q z_{l}\right) \prod_{k \neq j}\left(q x-q^{-1} y_{k}\right)}{x-y_{j}} .
$$

Thus, as pointed out by Foda et al., ${ }^{4}$ a KP tau function of the form (9) is hidden in $S(\boldsymbol{u}, \boldsymbol{v})$. Moreover, since the Bethe equations (38) imply the equations

$$
\begin{aligned}
\prod_{l=1}^{N}\left(y_{i}-z_{l}\right) \prod_{k \neq j}\left(q^{-1} y_{i}-q y_{k}\right) & \\
& =\prod_{l=1}^{N}\left(q^{-1} y_{i}-q z_{l}\right) \prod_{k \neq j}\left(q y_{i}-q^{-1} y_{k}\right) \quad(i=1, \ldots, n)
\end{aligned}
$$

for $y_{i}$ 's, the numerator of $f_{j}(x)$ can be factored out by the denominator $x-y_{j}$. Thus $f_{j}(x)$ 's turn out to be polynomials in $x$.

\section{Scalar product of states in models at $q=0$}

A class of solvable models, such as the phase model ${ }^{13}$ and and the totally asymmetric simple exclusion process (TASEP) model, ${ }^{26}$ can be formulated by a set of $2 \times 2 L$-matrices $L^{(l)}(u), l=1,2, \ldots, N$, and an $R$-matrix of the form

$$
R(u-v)=\left(\begin{array}{cccc}
f(u-v) & 0 & 0 & 0 \\
0 & 1 & g(u-v) & 0 \\
0 & g(u-v) & 0 & 0 \\
0 & 0 & 0 & f(u-v)
\end{array}\right)
$$

where

$$
f(u-v)=\frac{u^{2}}{u^{2}-v^{2}}, \quad g(u-v)=\frac{u v}{u^{2}-v^{2}} .
$$

This $R$-matrix is obtained as a "crystal" (namely, $q \rightarrow 0$ ) limit of the $R$ matrix of the 6 -vertex model and the XXZ spin chain. Unlike the XXZ chain of spin $1 / 2$, the $L$-matrices are not given by $2 \times 2$ blocks of the $R$-matrix and take a model-dependent form. We define the $T$-matrix as

$$
T(u) T(u)=\left(\begin{array}{cc}
A(u) & B(u) \\
C(u) & D(u)
\end{array}\right)=L^{(1)}(u) \cdots L^{(N)}(u)
$$


and consider the scalar product

$$
S_{n}(\boldsymbol{u}, \boldsymbol{v})=\left\langle 0\left|\prod_{i=1}^{n} C\left(u_{i}\right) \prod_{i=1}^{n} B\left(v_{i}\right)\right| 0\right\rangle .
$$

Remarkably, even if both $\boldsymbol{u}=\left(u_{1}, \ldots, u_{n}\right)$ and $\boldsymbol{v}=\left(v_{1}, \ldots, v_{n}\right)$ are free variables, the scalar product for those models has a determinant formula ${ }^{14}$ of the form

$$
S_{n}(\boldsymbol{u}, \boldsymbol{v})=\text { (simple factor) } \prod_{1 \leq i<j \leq n} \frac{u_{i} u_{j}}{u_{i}^{2}-u_{j}^{2}} \frac{v_{j} v_{i}}{v_{j}^{2}-v_{i}^{2}} \operatorname{det}\left(K_{i j}\right)_{i, j=1}^{n},
$$

where

$$
K_{i j}=\frac{\alpha\left(u_{i}\right) \delta\left(v_{j}\right)\left(v_{j} / u_{i}\right)^{n-1}-\delta\left(u_{i}\right) \alpha\left(v_{j}\right)\left(u_{i} / v_{j}\right)^{n-1}}{\left(u_{i}^{2}-v_{j}^{2}\right) / u_{i} v_{j}},
$$

$\alpha(u)$ and $\delta(u)$ being determined by the action of $A(u)$ and $D(u)$ on the pseudo-vacuum. If $\alpha(u)$ and $\delta(u)$ are given explicitly, we will be able to obtain a KP tau function (and hopefully a 2-KP tau function as well).

Such an interpretation can be found most clearly in the cases of the phase model ${ }^{27-29}$ and the 4 -vertex model, ${ }^{30}$ both of which are related to enumeration of boxed plane partitions. The scalar product of Bethe states in these cases becomes, up to a simple factor, a sum of products of two Schur functions:

$$
S(\boldsymbol{u}, \boldsymbol{v})=(\text { simple factor }) \sum_{\lambda \subseteq\left(N^{n}\right)} s_{\lambda}\left(u_{1}^{2}, \ldots, u_{n}^{2}\right) s_{\lambda}\left(v_{1}^{-2}, \ldots, v_{n}^{-2}\right) .
$$

By the same reasoning as the interpretation of (31), one can see that this sum is a tau function of the 2-KP hierarchy (or, rather, the 2D Toda hierarchy as Zuparic argued ${ }^{6}$ ) with respect to the time variables

$$
t_{n}=\frac{1}{n} \sum_{i=1}^{n} u_{i}^{2 n}, \quad \bar{t}_{n}=-\frac{1}{n} \sum_{i=1}^{n} v_{i}^{-2 n}
$$

Actually, this case admits yet another interpretation. In the course of deriving (44), the scalar product is shown to be a generating function for counting plane partitions in a box of size $n \times n \times N$. It is more or less well known ${ }^{16}$ that the Schur function $s_{\left(N^{n}\right)}$ gives such a generating function. Thus, in terms of this Schur function, the scalar product can be expressed as

$$
S(\boldsymbol{u}, \boldsymbol{v})=(\text { simple factor }) s_{\left(N^{n}\right)}\left(u_{1}^{2}, \ldots, u_{N}^{2}, v_{1}^{2}, \ldots, v_{N}^{2}\right) .
$$


This is similar to the formula (26) of the partition function of the 6 -vertex model for $q=e^{\pi / 3}$; one can thereby find an interpretation as a KP or 2-KP tau function with respect to the time variables

$$
t_{n}=\frac{1}{n} \sum_{i=1}^{n} u_{i}^{2 n}, \quad \bar{t}_{n}=\frac{1}{n} \sum_{i=1}^{n} v_{i}^{2 n} .
$$

\section{Acknowledgments}

The author thanks Omar Foda and Paul Zinn-Justin for useful comments and discussion. This work is partly supported by Grant-in-Aid for Scientific Research No. 19540179 and No. 21540218 from the Japan Society for the Promotion of Science.

\section{References}

1. M. Jimbo (ed.), "Yang-Baxter equations in Integrable Systems", World Scientific Publishing, 1990.

2. A. Zabrodin, Discrete Hirota's equation in integrable models, Int. J. Mod. Phys. B11 (1997), 3125-3158.

3. O. Foda, M. Wheeler and M. Zuparic, Domain wall partition functions and KP, J. Stat. Mech. (2009), P03017.

4. O. Foda, M. Wheeler and M. Zuparic, XXZ scalar products and KP, Nucl. Phys. B820 [FS] (2009), 649-663.

5. O. Foda and G. Schrader, XXZ scalar products, Miwa variables and discrete KP, arXiv:1003.2524.

6. M. Zuparic, Phase model expectation values and the 2-Toda hierarchy, J. Stat. Mech. (2009), P08010.

7. M. Zuparic, Studies in integrable quantum lattice models and classical hierarchies, arXiv:0908.3936.

8. V. Korepin, Calculation of norms of Bethe wave functions, Comm. Math. Phys. 86 (1982), 391-418.

9. A.G. Izergin, Partition function of the six-vertex model in a finite volume, Soviet Phys. Dokl. 32(1987), 878-879.

10. A.G. Izergin, D.A. Coker and V.E. Korepin, Determinant formula for the six vertex model, J. Phys. A: Math. Gen 25 (1991), 4315-4334.

11. N. Slavnov, Calculation of scalar products of wave functions and form factors in the framework of the algebraic Bethe ansatz, Theor. Math. Phys. 79 (1989), 502-508.

12. N. Kitanine, J. Maillet, V. Terras, Form Factors of the XXZ Heisenberg spin1/2 finite chain, Nucl. Phys. B554 (1999), 647-678.

13. N. Bogoliubov, A. Izergin and N. Kitanine, Correlation functions for a strongly correlated boson system, Nucl. Phys. B516 [FS] (1998), 501-528.

14. V.E. Korepin, N.M. Bogoliubov and A.G. Izergin, "Quantum inverse scattering method and correlation functions", Cambridge University Press, 1993. 
15. I. Macdonald, "Symmetric Functions and Hall Polynomials", Oxford University Press, USA, 1999.

16. P. Zinn-Justin, Six-vertex, loop and tiling models: Integrability and combinatorics, arXiv:0901.0665.

17. T. Miwa, M. Jimbo and E. Date, "Solitons", Cambridge University Press, 2000 .

18. T. Miwa, On Hirota's difference equation, Proc. Japan Acad., Ser. A, 58 (1982), 8-11.

19. M. Sato and Y. Sato, Soliton equations as dynamical systems on infinite dimensional Grassmann manifold, in "Nonlinear PDE in Applied Science" (Tokyo, 1992), Lecture Notes in Num. Appl. Anal. vol. 5 (1982), pp. 259 271.

20. K. Ueno and K. Takasaki, Toda Lattice Hierarchy, in "Group Representations and Systems of Differential Equations", Advanced Studies in Pure Mathematics vol. 4 (Kinokuniya, 1984), pp. 1-95.

21. K. Takasaki, Initial value problem for the Toda lattice hierarchy, in "Group Representations and Systems of Differential Equations", Advanced Studies in Pure Mathematics vol. 4 (Kinokuniya, 1984), pp. 139-163.

22. Yu. Stroganov, Izergin-Korepin determinant at a third root of unity, Theor. Math. Phys. 146 (2006), 53-62.

23. S. Okada, Enumeration of symmetry classes of alternating sign matrices and characters of classical groups, J. Algebraic Combin. 23 (2006), 43-69.

24. A.Yu. Orlov and T. Shiota, Schur function expansion for normal matrix model and associated discrete matrix models, Phys. Lett. A343 (2005), 384396.

25. V. Korepin and P. Zinn-Justin, Thermodynamic limit of the six-vertex model with domain wall boundary conditions, J. Phys. A: Math. Gen. 33 (2000), 7053-7066.

26. N.M. Bogoliubov, Determinantal representation of the time-dependent stationary correlation function for the totally asymmetric simple exclusion model, SIGMA 5 (2009), 052 (11 pages).

27. N. Bogoliubov, Boxed plane partitions as an exactly solvable boson model, J. Phys. A: Math. Gen. 38 (2005), 9415-9430.

28. K. Shigechi and M. Uchiyama, Boxed skew plane partitions and integrable phase model, J. Phys. A: Math. Gen. 38 (2005), 10287-10306.

29. N. Tsilevich, Quantum inverse scattering method for the q-boson model and symmetric functions, Funct. Anal. Appl. 40 (2006), 207-217.

30. N.M. Bogoliubov, Four-vertex models and random tilings, Theor. Math. Phys. 155 (2008), 523-535. 